\title{
Metastatic Malignant Neoplasm in the Extrahepatic Bile Ducts
}

National Cancer Institute

\section{Source}

National Cancer Institute. Metastatic Malignant Neoplasm in the Extrahepatic Bile Ducts. NCl Thesaurus. Code C96953.

A malignant neoplasm that has spread to the extrahepatic bile ducts from another anatomic site. Representative examples include metastatic melanoma and metastatic breast and gastric carcinomas. 